\title{
Estimation of Hospital Potential Capacity and Basic Reproduction Number
}

\author{
Fei Wang, ${ }^{1}$ Linhua Wang, ${ }^{2}$ and Peng Wang ${ }^{1}$ \\ ${ }^{1}$ Information Department, Southwest Hospital, Third Military Medical University, Chongqing 400038, China \\ ${ }^{2}$ Information Department, Daping Hospital, Third Military Medical University, Chongqing 400042, China \\ Correspondence should be addressed to Fei Wang; wangfxnyy@sina.com
}

Received 7 January 2014; Accepted 5 February 2014; Published 6 March 2014

Academic Editor: Weiming Wang

Copyright (C) 2014 Fei Wang et al. This is an open access article distributed under the Creative Commons Attribution License, which permits unrestricted use, distribution, and reproduction in any medium, provided the original work is properly cited.

In order to reflect the population covered by institutional medical services, the concept of hospital potential capacity is proposed and a formula for its estimation is developed based on a population dynamic model. Using the collected data on hospital outpatient and inpatient services and the demographical information on Chongqing as an example, the demand for medical resource allocation in Chongqing is dynamically estimated. Moreover, the proposed formula is also useful in the estimation of the basic reproduction number in epidemiology. The results can be contributed to the improvement of decision-making in the allocation of medical resources and the evaluation of the interventions and control efforts of the infectious disease.

\section{Introduction}

Hospital potential capacity reflects the population covered by the medical institutes, which is a crucial indicator to evaluate publicly served medical resource allocation. As the patient flow of a hospital is a complicated dynamic process, it is rather difficult to estimate the hospital potential capacity effectively by a simple statistical analysis of hospital outpatient and inpatient numbers.

Currently, there is no particular prototype for the estimation of hospital potential capacity, and most people are more concerned about the bed number or the bed capacity. However, bed planning and management are carried out under uncertainty and verification of environmental assessments and limited resources, and the spreadsheet calculations determined by simple planning and management capacity often underestimate the true demand for beds $[1,2]$. On the other hand, the dynamic capacity of hospitals can be determined to a certain extent by studying the patients' length of stay in hospitals, simulating relevant activities for patient flow in hospitals, and employing mathematical methods such as mixed exponential distributions, compartmental modelling, and simulation modelling to conduct integrated data analysis [3]. For example, the demand for beds and the waiting time for bed appointments can be estimated by analyzing a heart surgery patient's length of stay in an intensive care unit [4].

A new planning paradigm for improving the hospital capacity is to improve hospital resource allocation through the simulation of patient flow so as to avoid possible bottlenecks, which is also the new direction of research, and on which a growing number of research studies are focusing currently [5]. If the entry or the exit of patients under special medical conditions is considered as the patient flow and all the in-between activities or services require medical resources, this type of patient flow can be described as a resource network with basic network features. Hospital administrators can predict and assess the queuing model by determining the network features and, therefore, can improve the control of patient in-flows and enhance the resource utilization rate [6].

From a point of view of epidemiology, the basic reproduction number $R_{0}$ is commonly used to characterize disease transmissibility during an epidemic, which means the mean number of secondary cases of disease caused by a typical infected individual in a totally susceptible population in its lifetime without any control policies [7-9]. Now, many different methods have been proposed to estimate the basic reproduction number $R_{0}$ from the surveillance data [9-11]. 
For example, using the final epidemic size, [10] introduced the following calculation formula:

$$
R_{0}=\frac{N-1}{C} \sum_{i=S_{f}+1}^{S_{0}} \frac{1}{i}
$$

where $N$ is the population size, $C$ is the total number of cases in the given disease, and $S_{0}$ and $S_{f}$ are the numbers of susceptible individuals in the population at the start and end of the given disease, respectively. In addition, let $u_{0}=$ $S_{0} / N$ and $u_{\infty}=S_{f} / N$. Reference [12] obtained the following formula to estimate the basic reproduction number $R_{0}$ of the disease:

$$
R_{0}=\frac{1}{u_{0}-u_{\infty}}\left[\ln u_{0}-\ln u_{\infty}\right]
$$

Note that the parameter $N$ in (1) is equal to the hospital potential capacity to a certain extent. The estimation of the hospital potential capacity has very definite value in epidemiology.

In this study, by use of the surveillance data from Southwest Hospital and the demographic data of Chongqing city, we proposed a formula to estimate the hospital potential capacity. Chongqing is located in the southeast of inland China, on the middle-upper reaches of the Yangtze River, with a total population of more than 30 million. As a pilot zone for the national urban and rural comprehensive reform, Chongqing has a distinctive feature of large urban and rural areas with unbalanced economic and social developments, especially uneven distribution of medical resources. According to the statistics in 2012, there are 18 top-rated hospitals, among which six are large general hospitals with more than 1500 treatment tables, such as Southwest Hospital, the First Affiliated Hospital of Chongqing Medical University, Three Gorges Central Hospital, Xinqiao Hospital, Daping Hospital, and the Second Affiliated Hospital of Chongqing Medical University, mostly situated in the main city areas [13]. Southwest Hospital is the largest integrated medical and health institution, which provides medical treatment, education, and research facilities, accommodates more than 3000 beds with an average of over 10,000 outpatients per day and over 100,000 inpatients per year. Therefore, the study of population covered by the medical services offered by Southwest Hospital is regionally representative, and it provides important guidance to the allocation of medical resources in Chongqing.

This paper first constructs a population dynamics model, which reflects the hospital potential capacity on base of compartmental modeling, then utilizes the dynamic feature of the model and the data of the outpatients and inpatient numbers in Southwest Hospital from 2000 to 2012 to dynamically estimate the potential capacity for the same period. Based on the estimated potential capacity together with the surveillance data from the hospital on two common infectious diseases, viral hepatitis and brothers mouth disease, the corresponding basic reproduction number of these disease is dynamically estimated. By combining the annual demographic data in Chongqing for this time span, we dynamically estimate the annual demand of medical resource allocation in Chongqing

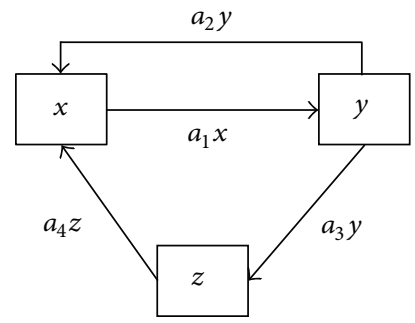

FIgURE 1: Flow chart of the population dynamic model.

from the estimated potential capacity. Considering the fact that Southwest Hospital is the largest medical institution in Chongqing (including staff, equipment, etc.), we collect further statistical data from another big general hospitalDaping Hospital-in order to adjust the data of Southwest Hospital. In this way, we can estimate the demand of annual medical resource allocation more reasonably and provide suggestions on the medical resource allocation in Chongqing, which enhance the decision-making in the allocation of medical resources to achieve the balance of demands-resource.

\section{Methods and Data}

2.1. Model Description. Intuitively, we divide the population into three types: healthy persons, outpatients, and inpatients. They are called the three compartments and are denoted by $x, y, z$, respectively. Generally, the population in the hospital service areas is relatively stable; therefore, the influences of birth and death in such areas are negligible. In other words, we can assume that the input and output of the population in this area remain the same. Hence, the population flow can be described as the following block diagram (Figure 1). Here $a_{1}$ represents the disease rate of healthy persons, $a_{2}$, the cure rate of outpatients, $a_{3}$, the hospitalizing rate of outpatients, and $a_{4}$, the cure rate of inpatients. According to the biological significance of the parameters, all $a_{i}, i=1,2,3,4$, are positive and $a_{2}+a_{3}=1$.

From Figure 1, we have the following differential equation model:

$$
\begin{gathered}
\frac{\mathrm{d} x}{\mathrm{~d} t}=-a_{1} x+a_{2} y+a_{4} z, \\
\frac{\mathrm{d} y}{\mathrm{~d} t}=a_{1} x-\left(a_{2}+a_{3}\right) y \\
\frac{\mathrm{d} z}{\mathrm{~d} t}=a_{3} y-a_{4} z
\end{gathered}
$$

Clearly, the population in a hospital service area is $N=x+$ $y+z$. According to model (3), we have $d N / d t=0$, which means that the hospital potential capacity remains constant. Note that $x=N-y-z$. Model (3) can be simplified as

$$
\begin{gathered}
\frac{\mathrm{d} y}{\mathrm{~d} t}=a_{1} N-\left(1+a_{1}\right) y-a_{1} z \triangleq F_{1}(y, z), \\
\frac{\mathrm{d} z}{\mathrm{~d} t}=a_{3} y-a_{4} z \triangleq F_{2}(y, z) .
\end{gathered}
$$

Here, we used $a_{2}+a_{3}=1$. 
2.2. Data. The demographic data of Chongqing is taken from the Chongqing Statistical Yearbook 2012 [14]. The hospital outpatient and inpatient data are extracted from the statistical reports of medical services offered by Southwest Hospital and Daping Hospital from 2000 to 2012. The data of viral hepatitis and hand-foot-and-mouth disease are extracted from Southwest Hospital between the years of 2003 and 2012. The sources of these data are valid and reliable, including the overall numbers of outpatients and inpatients. Among them, the data for Southwest Hospital are used for the dynamic estimation of potential capacity $N$ and the basic reproduction number $R_{0}$, whereas the data for Daping Hospital are mainly used for the estimation of percentages of outpatients from other hospitals in comparison to Southwest Hospital for more reasonable results in estimated demand of allocation of medical resources in Chongqing.

\section{Results}

3.1. Dynamics of Model (4). Let $F_{1}=0$ and $F_{2}=0$. It is easy to obtain a unique positive equilibrium $\left(y^{*}, z^{*}\right)$ in model (4), where

$$
y^{*}=\frac{a_{1} a_{4} N}{\left(1+a_{1}\right) a_{4}+a_{1} a_{3}}, \quad z^{*}=\frac{a_{1} a_{3} N}{\left(1+a_{1}\right) a_{4}+a_{1} a_{3}} .
$$

The Jacobian matrix $J$ of model (4) at the equilibrium is

$$
J=\left[\begin{array}{cc}
-\left(1+a_{1}\right) & -a_{1} \\
a_{3} & -a_{4}
\end{array}\right] .
$$

After a simple calculation, we find that the determinant of the Jacobian matrix $J$ is positive, and its trace is negative. Thus, the unique equilibrium $\left(y^{*}, z^{*}\right)$ is globally asymptotically stable based on the Routh-Hurwitz criterion and linearity of the model [15].

\subsection{Estimation of the Hospital Potential Capacity and the Basic} Reproduction Number. According to the dynamics of model (4), the hospital outpatients and inpatients tend towards the positive equilibrium (5). From the collected data for the monthly outpatients in Chongqing Southwest Hospital from 2000 to 2012, we see that the monthly outpatient numbers are relatively stable. Therefore, we use the number of annual outpatients for the estimation of $y^{*}$ at the equilibrium. We then assume that everyone gets sick at least once per year; that is, $a_{1}=1$. The annual parameters $a_{3}$ and $a_{4}$ can be estimated from the collected data. From the expression of $y^{*}$ in (5), we can determine the annual hospital potential capacity

$$
N=\frac{y^{*}\left(\left(1+a_{1}\right) a_{4}+a_{1} a_{3}\right)}{a_{1} a_{4}}
$$

Using formula (7) and the data from Southwest Hospital, we have the dynamical estimation of the potential capacity $N$ (corresponding to the no adjust term in Figure 2(a)). Based on formula (2) and the surveillance data of the viral hepatitis and hand-foot-and-mouth disease, the corresponding basic reproduction number $R_{0}$ of the diseases is further obtained
(Figure 3). From Figure 3, we can see that almost all basic reproduction numbers are greater than unity.

Furthermore, from the estimated annual potential capacity results and the annual demographic statistics of Chongqing, we can estimate the numbers of medical institutions required to meet the medical and health demand of population in Chongqing (corresponding to the no adjust term in Figure 2(b)). As seen from Figure 2, the population covered by the medical services of Southwest Hospital increases annually, and this rate of increase is much faster than the rate of increase in the population of Chongqing. Thus, the number of medical institutions required decreases each year.

3.3. Adjustment of the Number of Hospitals Required. In 2012, the medical and health demand of the whole population in Chongqing could be satisfied with six medical institutions of similar scale to Southwest Hospital (Figure 2(b)). However, there are 18 top-rated hospitals in the city [13]. Does it indicate that the present number of medical institutions is already saturated? Because the Southwest Hospital is the largest one in the region, no matter in the software or hardware facilities, but also the number of outpatients and inpatients compared with medical institutions. The collected data of the other large general hospital-Daping Hospital-suggest that the increasing rate of outpatients is still much slower than that of Southwest Hospital (Figure 4), with the average number of outpatients constituting only $45 \%$ that of Southwest Hospital. Thus, in this section, considering the imbalance of resources and service ability between different medical institutions, we adjust the annual number of outpatients of Southwest Hospital in order to obtain a better estimation of the annual demand of medical resources in Chongqing.

With respect to the statistical data of Daping Hospital, we consider $20 \%$ and $40 \%$ as correction coefficients in order to adjust the number of outpatients in Southwest Hospital while keeping the other parameters unchanged. Thus, we determine the hospital potential capacity and the number of medical institutions required using these coefficients, and these results are presented in Figure 2 (corresponding to the $20 \%$ and $40 \%$ adjust term in Figure 2).

\section{Discussion}

Because Southwest Hospital is the largest individual general medical institution in Chongqing, it has the largest annual outpatient and inpatient numbers. The results, presented in this paper and calculated from the dynamic analysis of data from Southwest Hospital, indicate the number of medical resource allocations for the same scale as that of Southwest Hospital. Under the same conditions, these numbers could be taken as the maximum number of allocations for medical resources. However, the data obtained from other large medical institutions mentioned in this paper cannot reach such level. We can assume that this sequence of data is in a descending order. Thus, in order to obtain realistic results, we adjust the data of Southwest Hospital using the correction coefficients. For instance, in 2012, the number of medical institutions required for a coefficient of $40 \%$ for Southwest 


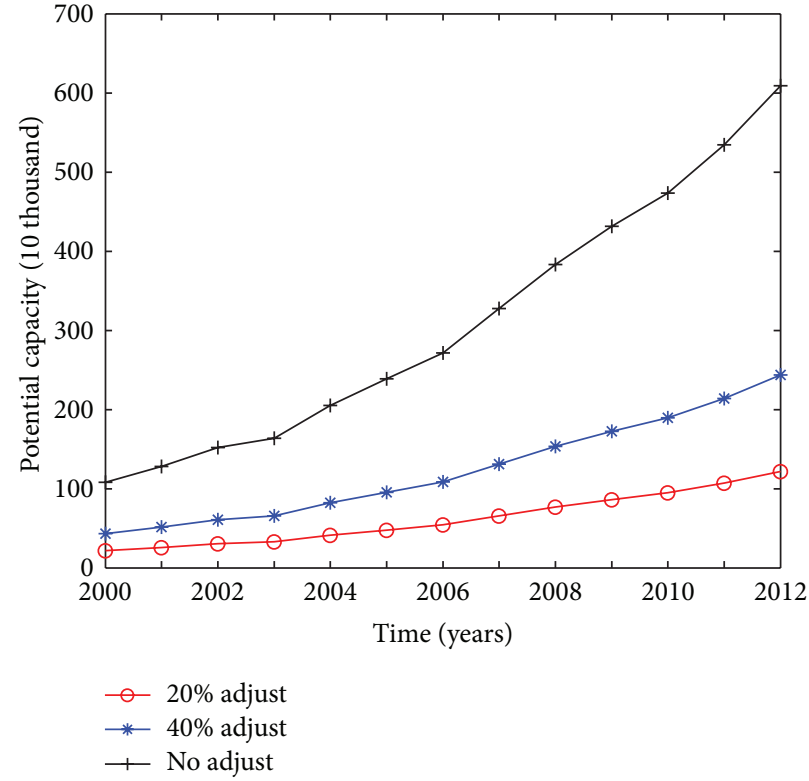

(a)

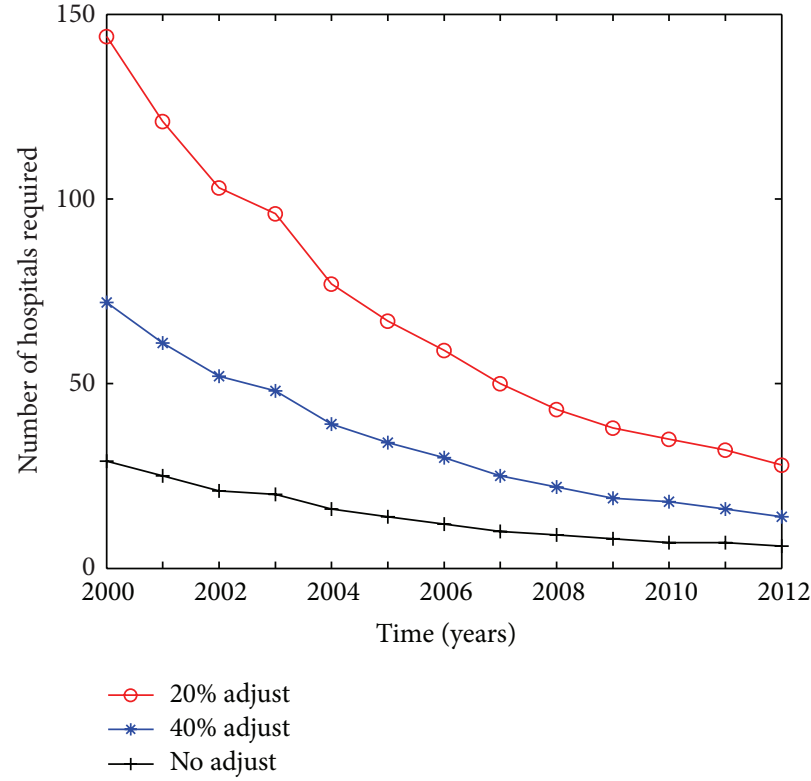

(b)

FIGURE 2: Illustration of the potential capacity and the number of hospital required under different correction coefficients. (a) Potential capacity, and (b) number of hospital required.

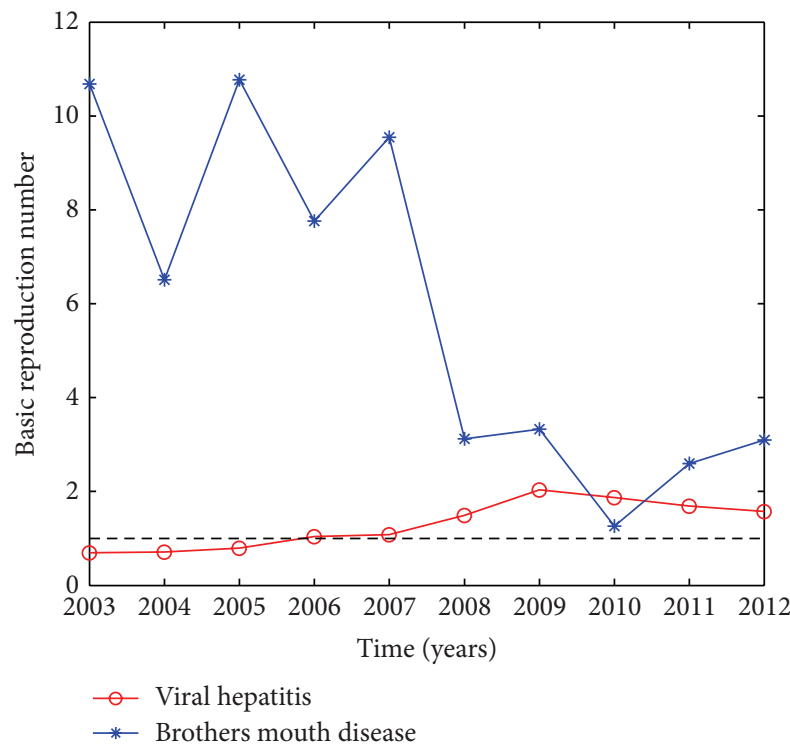

FIGURE 3: Illustration of the basic reproduction number for viral hepatitis and brothers mouth disease between the years of 2003 and 2012.

hospital is 14 , whereas only seven medical institutions in Chongqing, with a potential capacity of approximately 17.5 million, can meet or exceed this requirement. The number of medical institutions required for a coefficient of $20 \%$ for Southwest hospital is 28, whereas there are only 18 toprated hospitals in Chongqing with a potential capacity of about 22 million, which is much lower than the demand of the total population of more than 30 million. Therefore, we

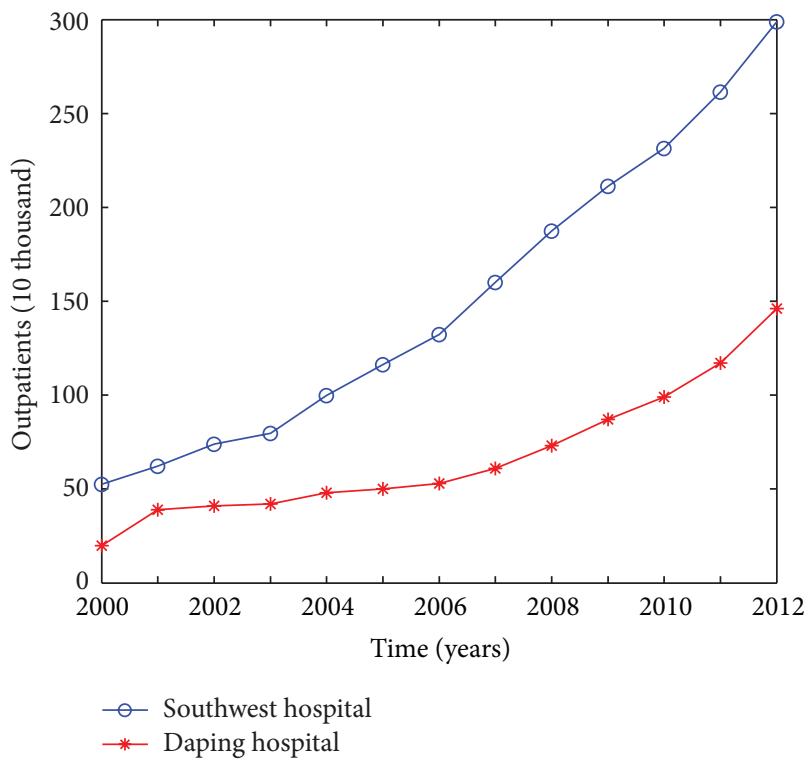

Figure 4: Comparison of outpatients in Chongqing Southwest Hospital and Chongqing Daping Hospital.

conclude that the existing medical institutions in Chongqing still cannot meet the demand of the whole population.

Regarding the estimation of the basic reproduction number $R_{0}$, such as formula (1) or (2), we can use the proposed formula (7) to estimate the requisite population size. Thus, it is a meaningful study to combine the formulae (2), (7) with clinical surveillance data during an epidemic to obtain the estimation of the basic reproduction number. For two 
common infectious diseases, such as viral hepatitis and handfoot-and-mouth disease, the estimated results indicate that almost all $R_{0}$ are greater than unity. Note that $R_{0}>1$ means that the disease is endemic in the area. Therefore, the incidence will not be significant in the near future with the current status. This will help us to understand the trend of the epidemic and has potential benefits in evaluating different interventions and control efforts $[16,17]$. Our conclusion shows $R_{0}$ is generally larger as the infectious disease is caused by the enteric viruses, and happens to children. More stricter and effective control efforts are necessary in control of the epidemic. In order to control the epidemic, we need to take stricter and more effective control efforts.

\section{Conflict of Interests}

The authors declare that there is no conflict of interests regarding the publication of this paper.

\section{Acknowledgment}

This work is partially supported by the National High-tech Research and Development Program (863 Program) (no. 2012AA02A616).

\section{References}

[1] J. Appleby, “The hospital bed: on its way out?” British Medical Journal, vol. 346, no. 11, p. f1563, 2013.

[2] P. R. Harper and A. K. Shahani, "Modelling for the planning and management of bed capacities in hospitals," Journal of the Operational Research Society, vol. 53, no. 1, pp. 11-18, 2002.

[3] A. Marshall, C. Vasilakis, and E. El-Darzi, "Length of staybased patient flow models: recent developments and future directions," Health Care Management Science, vol. 8, no. 3, pp. 213-220, 2005.

[4] S. Gallivan, M. Utley, T. Treasure, and O. Valencia, "Booked inpatient admissions and hospital capacity: mathematical modelling study," British Medical Journal, vol. 324, no. 5, pp. 280-282, 2002.

[5] B. Rechel, S. Wright, J. Barlow, and M. McKee, "Hospital capacity planning: from measuring stocks to modelling flows," Bulletin of the World Health Organization, vol. 88, no. 8, pp. 632636, 2010.

[6] M. J. Côté, "Understanding patient flow," Decision Line, vol. 31, no. 1, pp. 8-10, 2000.

[7] O. Diekmann, J. A. Heesterbeek, and J. A. Metz, "On the definition and the computation of the basic reproduction ratio $\mathrm{R} 0$ in models for infectious diseases in heterogeneous populations," Journal of Mathematical Biology, vol. 28, no. 4, pp. 365-382, 1990.

[8] P. van den Driessche and J. Watmough, "Reproduction numbers and sub-threshold endemic equilibria for compartmental models of disease transmission," Mathematical Biosciences, vol. 180, pp. 29-48, 2002.

[9] A. Cori, N. M. Ferguson, C. Fraser, and S. Cauchemez, "A new framework and software to estimate time-varying reproduction numbers during epidemics," The American Journal of Epidemiology, vol. 178, no. 9, pp. 1505-1512, 2013.
[10] E. Vynnycky, A. Trindall, and P. Mangtani, "Estimates of the reproduction numbers of Spanish influenza using morbidity data," International Journal of Epidemiology, vol. 36, no. 4, pp. 881-889, 2007.

[11] H. F. Huo, S. J. Dang, and Y. N. Li, "Stability of a two-strain tuberculosis model with general contact rate," Abstract and Applied Analysis, vol. 2010, Article ID 293747, 31 pages, 2010.

[12] S. Liao, W. Yang, and X. Chen, "The basic reproduction number for the cholera outbreak," Journal on Numerical Methods and Computer Applications, vol. 33, no. 3, pp. 189-197, 2012.

[13] Health and Family Planning Commission of Chongqing, http:// www.cqwsj.gov.cn/jyzn/yyxx/2012-8/10653.html.

[14] Chongqing Municipal Bureau of Statistics, http://www.cqtj.gov .cn/tjnj/2012/indexch.htm.

[15] J. Y. Zhang and B. Y. Feng, Geometric Theory of Ordinary Differential Equations and Bifurcation Problems, Peking University Press, Beijing, China, 2000 (Chinese).

[16] C. Fraser, S. Riley, R. M. Anderson, and N. M. Ferguson, "Factors that make an infectious disease outbreak controllable," Proceedings of the National Academy of Sciences of the United States of America, vol. 101, no. 16, pp. 6146-6151, 2004.

[17] N. M. Ferguson, D. A. T. Cummings, C. Fraser, J. C. Cajka, P. C. Cooley, and D. S. Burke, "Strategies for mitigating an influenza pandemic," Nature, vol. 442, no. 7101, pp. 448-452, 2006. 


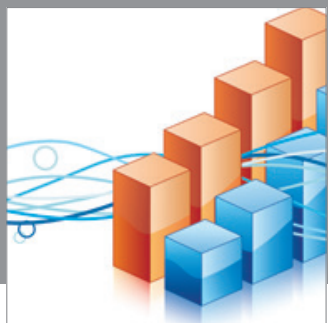

Advances in

Operations Research

mansans

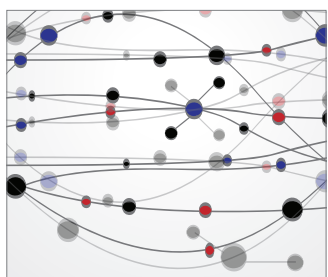

The Scientific World Journal
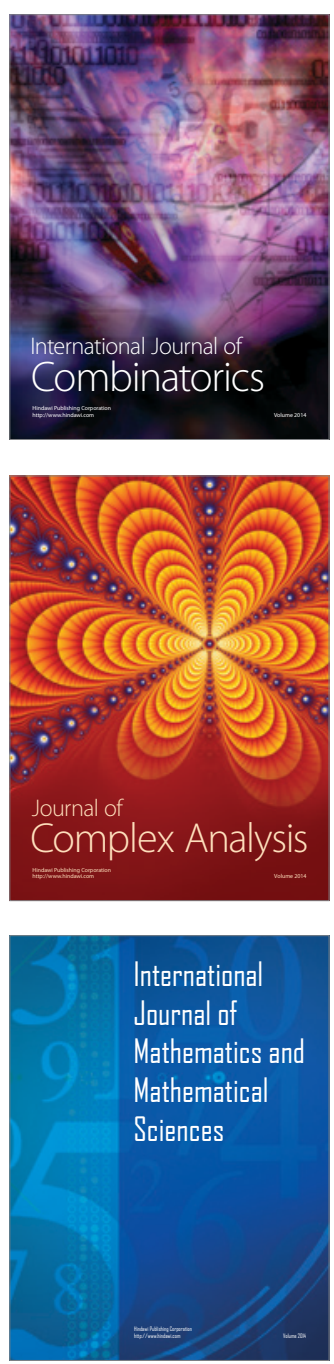
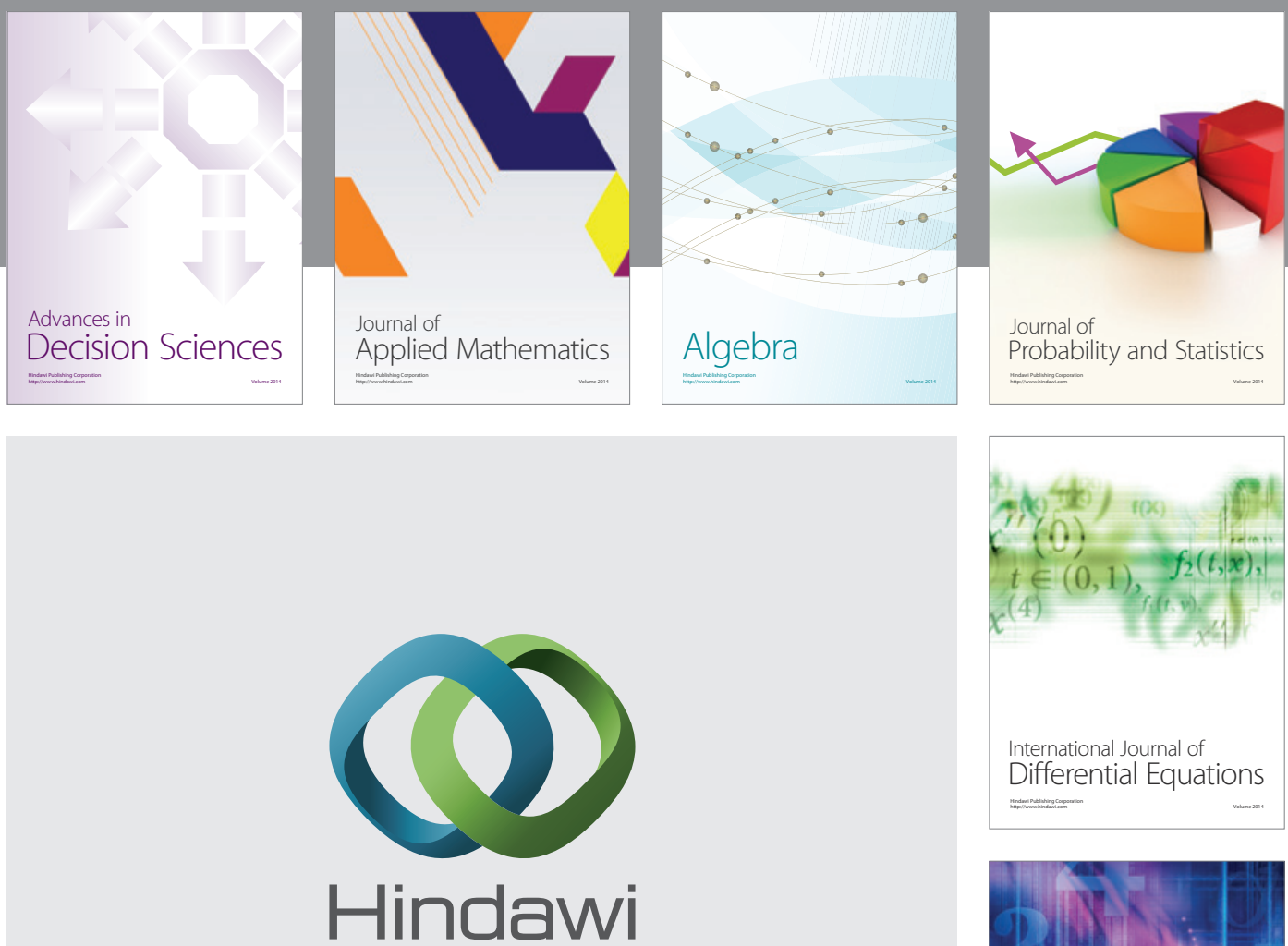

Submit your manuscripts at http://www.hindawi.com
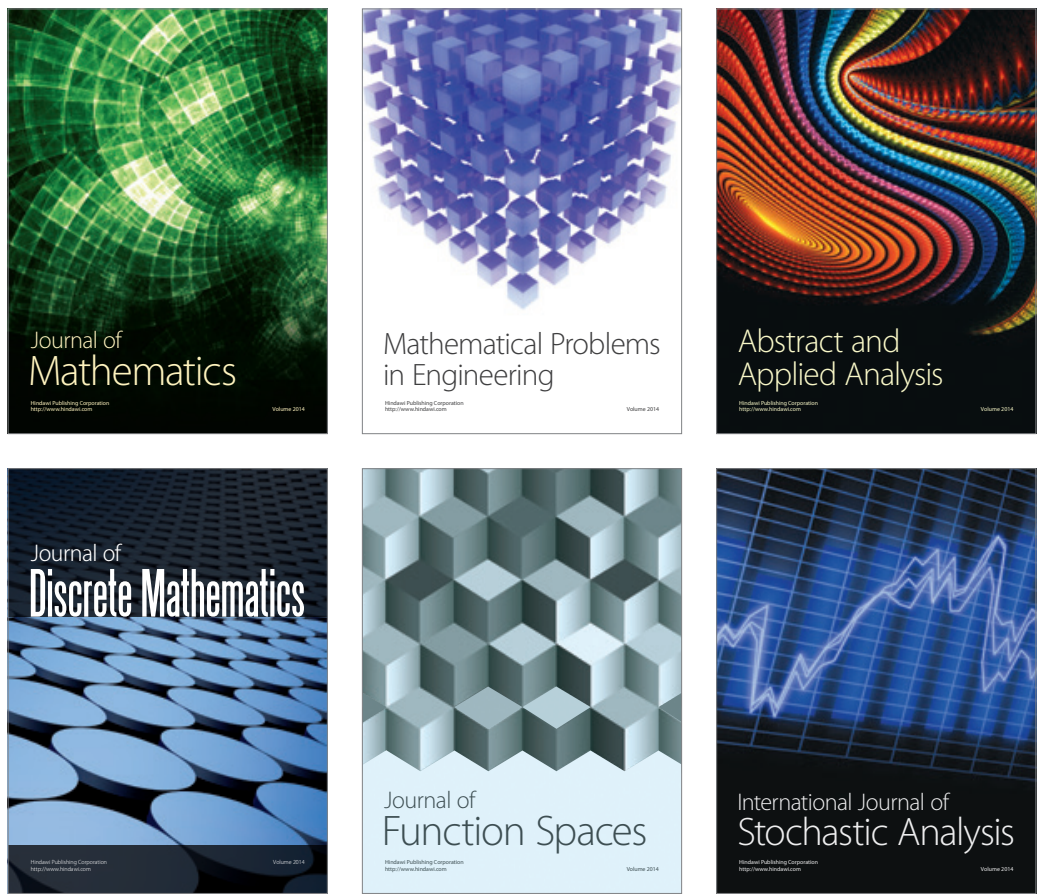

Journal of

Function Spaces

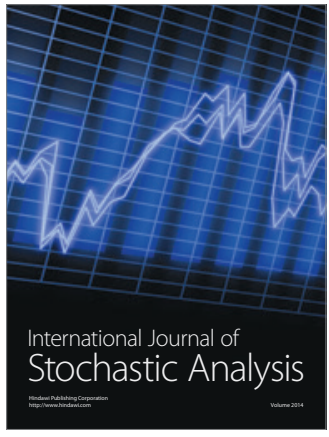

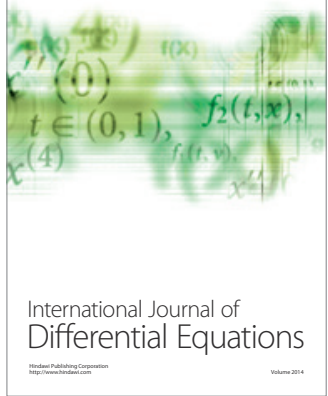
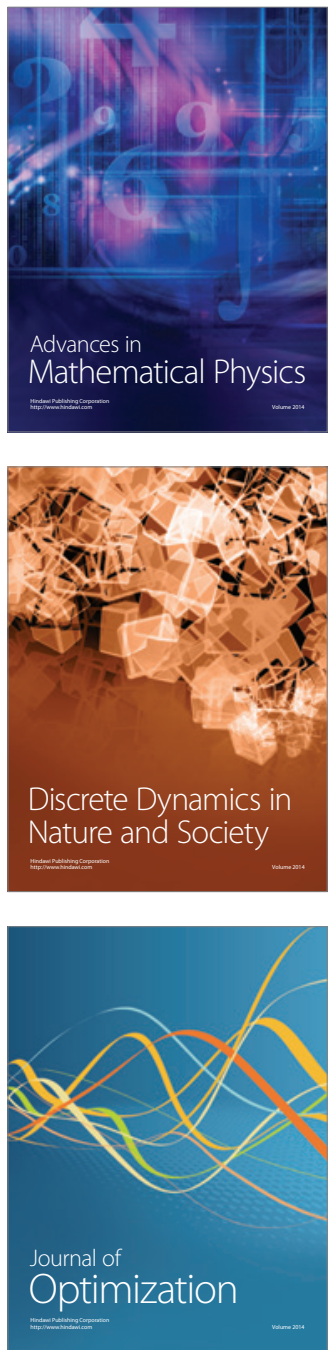